\title{
High power gain for stimulated Raman amplification in $\mathrm{CuAIS}_{\mathbf{2}}$
}

B. H. Bairamov, A. Aydinli, I. V. Bodnar', Yu. V. Rud', V. K. Nogoduyko, and V. V. Toporov

Citation: Journal of Applied Physics 80, 5564 (1996); doi: 10.1063/1.363820

View online: https://doi.org/10.1063/1.363820

View Table of Contents: http://aip.scitation.org/toc/jap/80/10

Published by the American Institute of Physics

\section{Articles you may be interested in}

Chemistry of defect induced photoluminescence in chalcopyrites: The case of $\mathrm{CuAlS}_{2}$ Journal of Applied Physics 109, 023519 (2011); 10.1063/1.3544206

Band structure, Born effective charges, and lattice dynamics of $\mathrm{CuInS}_{2}$ from ab initio calculations The Journal of Chemical Physics 117, 2726 (2002); 10.1063/1.1489993

Optical properties of $\mathrm{CuAlSe}{ }_{2}$

Journal of Applied Physics 88, 1923 (2000); 10.1063/1.1305858

Photoluminescence studies in CuAlSe 2 epilayers grown by low-pressure metalorganic chemical-vapor deposition

Journal of Applied Physics 77, 1225 (1995); 10.1063/1.358990

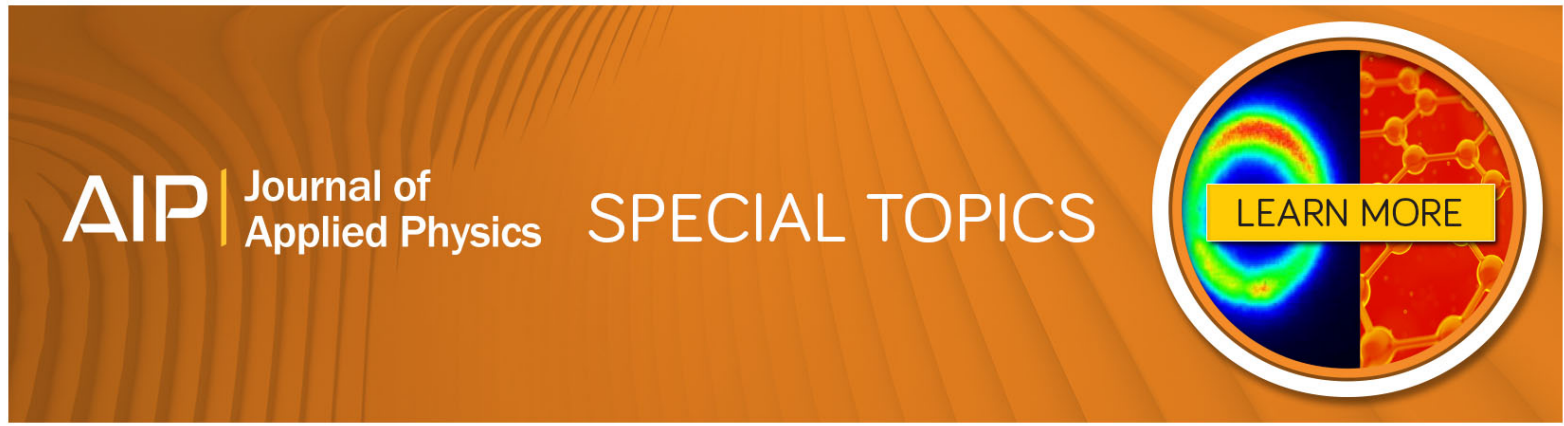




\title{
High power gain for stimulated Raman amplification in CuAIS ${ }_{2}$
}

\author{
B. H. Bairamov a) and A. Aydinli \\ Bilkent University, Department of Physics, Bilkent, Ankara, 06533 Turkey \\ I. V. Bodnar', Yu. V. Rud', V. K. Nogoduyko, and V. V. Toporov \\ A. F. Ioffe Physico-Technic Institute, Russian Academy of Sciences, 194021 Russia
}

(Received 22 February 1996; accepted for publication 11 July 1996)

\begin{abstract}
The spontaneous Raman spectra of the chalcopyrite structure crystal $\mathrm{CuAlS}_{2}$, which is promising for nonlinear optical applications, has been investigated at 8 and $300 \mathrm{~K}$. The main aim of this study is to compare the absolute spontaneous Raman scattering efficiency in $\mathrm{CuAlS}_{2}$ crystals with that of their isomorphous analog, zinc-blende structure GaP crystals, known as one of the most efficient materials for Raman amplification. Observation of a high value of absolute scattering efficiency $S / L d \Omega$ (where $S$ is the fraction of incident power that scatters into the solid angle $d \Omega$ and $L$ is the optical path length with $S / L d \Omega=9.5 \times 10^{-5} \mathrm{~cm}^{-1} \mathrm{sr}^{-1}$ ), together with relatively narrow linewidth $\left(\Gamma=5.1 \mathrm{~cm}^{-1}\right.$, full width at half maximum at room temperature and $\Gamma=1.5 \mathrm{~cm}^{-1}$ at $8 \mathrm{~K}$ for the strongest $\Gamma_{1}$ phonon mode of $\mathrm{CuAlS}_{2}$ at $314 \mathrm{~cm}^{-1}$ ) indicate that $\mathrm{CuAlS}_{2}$ has the highest value of the stimulated Raman gain coefficient $g_{s} / I$ where $I$ is the incident laser power density. The calculated value of this gain is $g_{s} / I=2.1 \times 10^{-6} \mathrm{~cm}^{-1} / \mathrm{W}$ at $300 \mathrm{~K}$ and $5.0 \times 10^{-6} \mathrm{~cm} / \mathrm{W}$, at $8 \mathrm{~K}$ for $514.5 \mathrm{~nm}$ laser excitation, and is larger than those for the appropriate vibrational modes of various materials (including $\mathrm{GaP}, \mathrm{LiNbO}_{3}, \mathrm{Ba}_{2} \mathrm{NbO}_{5} \mathrm{O}_{15}, \mathrm{CS}_{2}$, and $\mathrm{H}_{2}$ ) investigated so far. The calculations show that cw Raman oscillator operation in $\mathrm{CuAlS}_{2}$ is feasible with low power threshold of pump laser. (C) 1996 American Institute of Physics. [S0021-8979(96)03020-4]
\end{abstract}

\section{INTRODUCTION}

Recently, a great deal of interest has been focused on the growth of the ternary I-III- $\mathrm{VI}_{2}$ and II $-\mathrm{IV}-\mathrm{V}_{2}$ semiconductor compounds which crystallize in the chalcopyrite $\left(\mathrm{CuFeS}_{2}\right)$-type structure. Within this family there are many ternary compounds which have a band gap of in the range of $3.5-1.0 \mathrm{eV}$ covering the wide spectral region from ultraviolet to near infrared and having different attractive linear and nonlinear optical properties. The usefulness of these compounds as nonlinear optical components owing to their high nonlinear susceptibilities (which are comparable to or even greater than those of their many binary zinc-blende structure analogs) and especially to the uniaxial birefringence (which makes it possible for phase matching), is well known.

For example, zinc germanium diphosphide $\left(\mathrm{ZnGeP}_{2}\right)$ and cadmium germanium diarsenide $\left(\mathrm{CdGeAs}_{2}\right)$ crystals are promising candidates for efficient second harmonic generators ${ }^{1}$ as well as mid-infrared optical parametric oscillators ${ }^{2,3}$ exhibiting very high energy conversion efficiencies (up to $46 \%$ ), at moderate average power levels. ${ }^{4}$ Another famous member of this family is silver gallium disulfide $\left(\mathrm{AgGaS}_{2}\right)$ with $E_{g}=2.7 \mathrm{eV}$ (at room temperature) which is an important material due to its large nonlinear optical coefficients and birefringence. ${ }^{5}$

Due to the observation of the direct band gap for these compounds ${ }^{6}$ with chalcopyrite-type structure, one can expect their potential applications for optoelectronic devices such as light emitting diodes and laser diodes operating in the blue and ultraviolet wavelength region.

The chalcopyrite-type semiconductor copper indium diselenide $\left(\mathrm{CuInSe}_{2}\right)$ has $E_{g}=1.0 \mathrm{eV}$ (at room temperature)

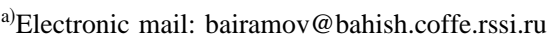

which is in the energy range for optimum solar energy conversion with the absorption coefficient of about $5 \times 10^{-5}$ $\mathrm{cm}^{-1}$ (near the band gap for polycrystalline thin films) which is the highest value reported for any semiconductor up to now. ${ }^{7-9}$

In addition, device applications of chalcopyrite-type semiconductor compounds through the formation of efficient near lattice matched heterojunctions with the semiconductor III-V and II-VI compounds is also under consideration.

As mentioned previously, the chalcopyrite-structure compounds are close isoelectronic analogs of zinc-blende structure binary semiconductors where replacement of cationic sublattice by different atomic species induces a change from $T_{d}^{2}(F \overline{43} m)$, characteristic of zinc-blende structure to the tetragonal space group $D_{2 d}^{12}(I \overline{4} 2 d)$ characteristic for chalcopyrite structure with so-called weak tetrahedral distortion and tetragonal compression of anions with respect to the ideal zinc-blende structure. Therefore, the vibrational and electrical properties of chalcopyrite-type compounds have striking analogues with their II-VI and III-V homologies.

One of the promising chalcopyrite-type semiconductors for nonlinear optical applications is copper aluminum disulfide $\left(\mathrm{CuAlS}_{2}\right)$, which has a direct gap of $3.49 \mathrm{eV}$ at room temperature, the widest value among other chalcopyrite compounds. Recent progress in the growth of undoped and doped bulk $^{10}$ as well as the heteroepitaxial growth of $\mathrm{CuAlS}_{2}$ layers on (100) GaP and (100) GaAs substrates by metalorganic vapor phase epitaxy ${ }^{11}$ and molecular beam epitaxy ${ }^{12}$ makes them even more technologically promising.

As a matter of fact, the nature and origin of the high nonlinear optical susceptibility and Raman scattering efficiency are closely interrelated. It should be pointed out that the nonlinear optical properties of the insulating crystals are derived from both: first, the perturbation of the optical polar- 
TABLE I. Comparison of frequencies $\nu$ (in $\mathrm{cm}^{-1}$ ) of the Raman and infrared active zone center optical phonons in $\mathrm{CuAlS}_{2}$ with their symmetry assignment and zinc-blend origin. The splitting of polar modes with nonzero dipole moments into transverse and longitudinal modes is also shown.

\begin{tabular}{|c|c|c|c|c|c|}
\hline \multirow{2}{*}{$\begin{array}{l}\text { Chalcopyrite } \\
\text { mode } \\
\text { symmetry }\end{array}$} & \multirow{2}{*}{$\begin{array}{l}\text { Zinc-blende } \\
\text { mode } \\
\text { symmetry }\end{array}$} & \multirow{2}{*}{$\begin{array}{c}\text { Raman } \\
\text { scat. }^{\mathrm{a}, \mathrm{b}} \\
T=300 \mathrm{~K}\end{array}$} & \multirow{2}{*}{$\begin{array}{c}\text { Infrared } \\
\text { reflectivity } \\
\text { /absorption } \\
\quad T=300 \mathrm{~K}\end{array}$} & \multicolumn{2}{|c|}{$\begin{array}{l}\text { Raman scat. } \\
\text { present work }\end{array}$} \\
\hline & & & & $T=300 \mathrm{~K}$ & $T=8 \mathrm{~K}$ \\
\hline$\Gamma_{5}(L, T)$ & $\left(X_{5}\right)_{\mathrm{ac}}$ & $76 / 76$ & & $75.5 / 74.0$ & 77.4 \\
\hline$\Gamma_{3}$ & $\left(W_{2}\right)_{\mathrm{ac}}$ & 98 & & 96 & $\ldots$ \\
\hline$\Gamma_{4}(L, T)$ & $\left(W_{2}\right)_{\mathrm{ac}}$ & $112 / 112$ & & 108 & $\ldots$ \\
\hline$\Gamma_{5}(L, T)$ & $\left(W_{4}\right)_{\mathrm{nc}}$ & $137 / 137$ & & 135 & $\cdots$ \\
\hline$\Gamma_{5}(L, T)$ & $\left(W_{3}\right)$ & $217 / 216$ & $217 / 216$ & $217.5 / 217.5$ & 219.5 \\
\hline$\Gamma_{3}$ & $\left(X_{3}\right)$ & 268 & & 263 & 266.5 \\
\hline$\Gamma_{5}(L, T)$ & $\left(X_{5}\right)_{\mathrm{opt}}$ & $266 / 263$ & $266 / 263$ & $263 / 261$ & $275 / 265$ \\
\hline$\Gamma_{4}(L, T)$ & $\left(W_{2}\right)_{\mathrm{opt}}$ & $284 / 271$ & $284 / 271$ & $-/ 272$ & $-/ 274$ \\
\hline$\Gamma_{1}$ & $\left(W_{1}\right)$ & 315 & & 314 & 317 \\
\hline$\Gamma_{5}(L, T)$ & $\left(W_{4}\right)_{\mathrm{opt}}$ & $-/ 432$ & $-/ 432$ & $\cdots$ & $\cdots$ \\
\hline$\Gamma_{3}$ & $\left(W_{2}\right)_{\mathrm{opt}}$ & 443 & & 442 & 448 \\
\hline$\Gamma_{5}(L, T)$ & $\Gamma_{15}$ & $497 / 444$ & $497 / 444$ & $496 / 444$ & $498 / 449$ \\
\hline$\Gamma_{4}(L, T)$ & $\Gamma_{15}$ & $498 / 446$ & $498 / 446$ & $498 / 446$ & $503 / 448$ \\
\hline \multirow{3}{*}{$\begin{array}{l}\text { Second-order } \\
\text { features }\end{array}$} & & & & 123 & $\ldots$ \\
\hline & & & & 372 & $\cdots$ \\
\hline & & & & 553 & $\ldots$ \\
\hline
\end{tabular}

${ }^{a}$ Reference 21.

${ }^{b}$ Reference 22.

izability by an electromagnetic field acting through a lattice displacement-so-called lattice or deformation potential interaction and second, the perturbation of the optical polarizability produced by the direct action of the field on the electronic levels-so-called nonlattice or electro-optic interaction. The same interactions manifest themselves in the process of spontaneous Raman scattering by lattice vibrations.

For piezoelectric crystal structures, which lack a center of inversion, Loudon ${ }^{13}$ has showed that both deformation potential and electro-optic interactions contribute to the Raman scattering efficiency for zone center longitudinal optical $[\mathrm{LO}(\Gamma)]$ modes, but that only the lattice interaction contributes to the scattering efficiency for transverse optical $[\mathrm{TO}(\Gamma)]$ modes. Therefore, the measurements of absolute scattering efficiency and linewidth of the phonon modes can permit a determination of available power gain coefficient for stimulated Raman scattering amplification.

Previously, the high value of absolute scattering efficiency for the $\mathrm{LO}(\Gamma)$ phonon from spontaneous Raman scattering intensity measurements of binary undoped $\mathrm{GaP}^{14}$ has been observed. This, together with the observed anomalously narrow spectral linewidth, indicates a large gain coefficient for stimulated Raman scattering. The measured value of this gain was quite large by comparison with other materials, and a low threshold of pump laser power had been predicted. ${ }^{15}$

Recently, the low threshold pump power Raman laser based on GaP and suitable for applications in optical communication systems in terahertz frequency region has been demonstrated. ${ }^{16,17}$ In this connection, the knowledge of the absolute spontaneous Raman scattering efficiency and gain coefficient for stimulated Raman scattering of $\mathrm{CuAlS}_{2}$ crystals and their direct comparison with those of GaP crystals is of considerable interest. To our knowledge those measure- ments for $\mathrm{CuAlS}_{2}$ have not been reported so far.

In the present study, an attempt has been made to find out the relationship between the spontaneous scattering intensity of $\mathrm{CuAlS}_{2}$ and GaP crystals with the main aim of determining the nonlinear Raman power gain amplification for the strongest phonon modes of $\mathrm{CuAlS}_{2}$ for stimulated Raman scattering process. It is found that this gain exceeds those reported for any other promising materials widely used for stimulated Raman scattering operation.

\section{EXPERIMENTAL PROCEDURES}

The single crystal samples used in this experiment were grown by the chemical vapor transport method in an evacuated quartz tube using iodine as a transport agent followed by directional freezing. The starting polycrystalline $\mathrm{CuAlS}_{2}$ compounds were prepared by direct melting of the constituent elements of high quality in stoichiometric amounts. The $\mathrm{CuAlS}_{2}$ crystals were light green in color. Typical morphology of platelike crystals showed a well developed surface along [111] axis, with [101] and [112] facets, which are parallel to the axis. The typical dimensions of the grown crystals were about $5 \times 3 \times 0.4 \mathrm{~mm}^{3}$.

The crystallographic parameters obtained on our samples by using x-ray diffraction techniques carried out at room temperature with Debye-Scherrer powder method confirmed that a single phase growth with chalcopyrite structure is achieved with $a=5.3336 \AA$ and $c=10.440 \AA$. These values are in good agreement with the parameters previously reported by Brandt et al. ${ }^{18}$

The measurements of spontaneous Raman scattering spectra were performed in the backscattering configuration for crossed and parallel polarization of incident and scattered light from the naturally grown [112] plane, which corre- 


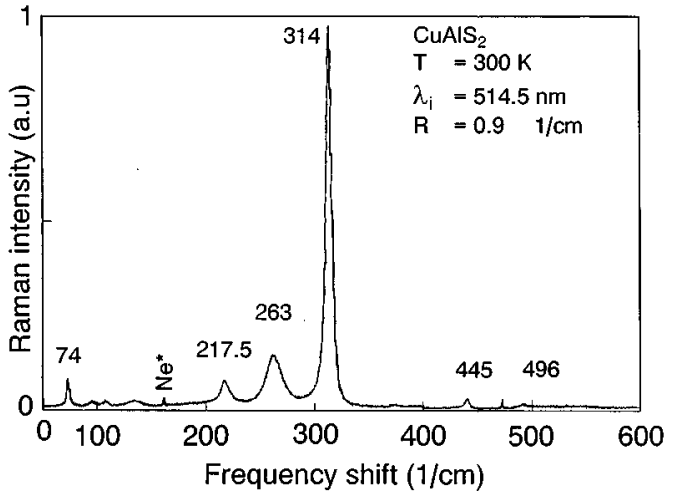

FIG. 1. The overall Raman spectrum of $\mathrm{CuAlS}_{2}$ obtained at room temperature in the backscattering configuration from (112) plane for parallel polarizations of incident and scattered light. The excitation wavelength is 514.5 $\mathrm{nm}$ and spectral resolution $0.9 \mathrm{~cm}^{-1}$. Notice the large intensity of $\Gamma_{1}$ phonon mode at $314 \mathrm{~cm}^{-1}$. Small peak at $329 \mathrm{~cm}^{-1}$ is from Ne reference lamp.

sponds to the (111) plane of zinc-blende structure material. Both 514.5 and $476.5 \mathrm{~nm}$ lines of an $\mathrm{Ar}^{+}$laser, which are far from resonance with $E_{g}$ of $\mathrm{CuAlS}_{2}$ were used as the excitation sources. The scattered light was analyzed using a JobinYvon U 1000 double grating monochromator with a spectral resolution of $0.9-1.1 \mathrm{~cm}^{-1}$ and detected with a cooled GaAs photomultiplier operating in the photon counting mode. All measurements were performed at room temperature and at 8 $\mathrm{K}$ using a closed-cycle cryostat.

For the measurement of absolute scattering efficiency, we used a zinc-blende structure semi-insulating (si) $\mathrm{GaP}$ crystal oriented along (111) direction. Backscattering measurement from the (111) face performed under the same experimental conditions as from $\mathrm{CuAlS}_{2}$ crystal. For absolute frequency calibration, we used a Ne spectral lamp.

\section{RESULTS AND DISCUSSION}

As already said, the $\mathrm{A}^{\mathrm{I}} \mathrm{B}^{\mathrm{III}} \mathrm{C}^{\mathrm{VI}}{ }_{2}$ chalcopyrite structure has the symmetry $D_{2 d}^{12}(I \overline{4} 2 d)$ and the body centered tetragonal unit cell. For ideal chalcopyrite crystals the long dimension $c$ must be twice the cubic length $a$. In our case, the structural

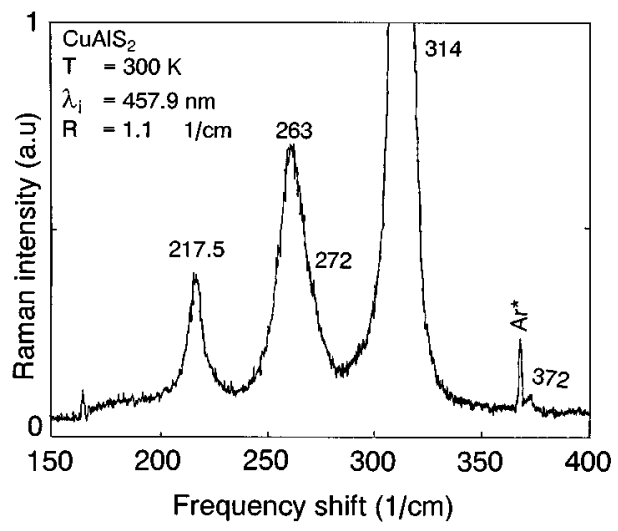

FIG. 2. Raman spectrum of $\mathrm{CuAlS}_{2}$ obtained at room temperature in the backscattering configuration from (112) plane for parallel polarizations of incident and scattered light in expanded scale in the range $150-400 \mathrm{~cm}^{-1}$. The excitation wavelength is $457.9 \mathrm{~nm}$ and spectral resolution $1.1 \mathrm{~cm}^{-1}$.

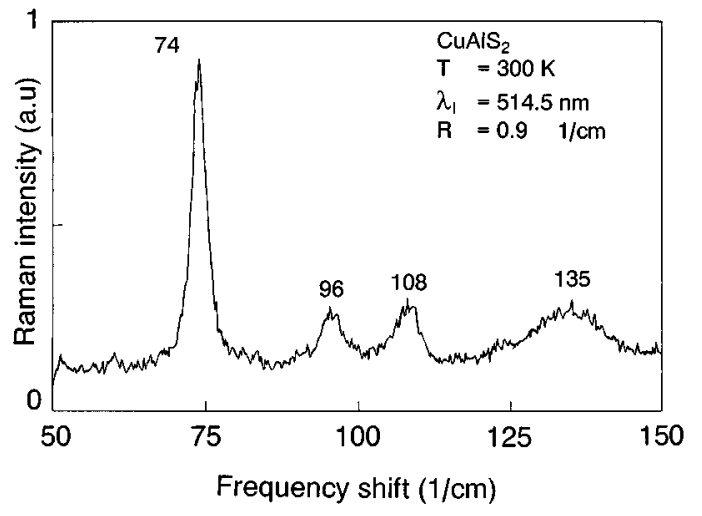

FIG. 3. Raman spectrum of $\mathrm{CuAlS}_{2}$ obtained at room temperature in the backscattering configuration from (112) plane in the expanded scale for parallel polarizations of incident and scattered light. The excitation wavelength is $514.5 \mathrm{~nm}$ and spectral resolution is $0.9 \mathrm{~cm}^{-1}$.

parameter is $\eta=(c / 2 a)=0.980$ This value is very close to $\eta=1$ among all chalcopyrite-type compounds. The mean tetrahedral distortion of anions with respect to ideal zinc-blende position changes the first-nearest-neighbor interatomic distances $d_{\mathrm{AC}}$ and $d_{\mathrm{AB}}$ which can be characterized by parameter $u$ given by

$$
n-\frac{1}{4}=\left(d_{\mathrm{AC}}^{2}-d_{\mathrm{BC}}^{2}\right) a^{2} \text {. }
$$

For $\mathrm{CuAlS}_{2}, d_{\mathrm{AC}}=2.351 \AA$ and $d_{\mathrm{BC}}=2.239 \AA$ and $u=0.2547 \AA$. For comparison, analogous parameters for other chalcopyrite-type compounds are $\eta=1.004, u=0.224$ $\AA$ for $\mathrm{CuInSe}_{2}{ }^{19}$ and $\eta=0.896, u=0.27$ for $\mathrm{AgGaSe}_{2}{ }^{20}$ which indicate much larger structure modifications than $\mathrm{CuAlS}_{2}$. Due to the fact that there are two different types of cations in chalcopyrite structure compounds and because their unit cell volume is four times smaller than in a typical zinc-blende material, there is a four-to-one correspondence between the Brillouin zones of the chalcopyrite and zinc-blende structure compounds. Therefore, the chalcopyrite structure semiconductors may be regarded as a superlattice of the zinc-blende-

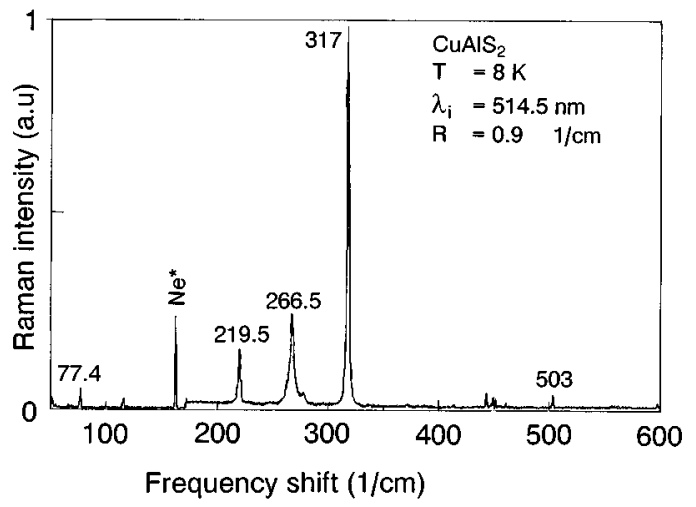

FIG. 4. The overall Raman spectrum of $\mathrm{CuAlS}_{2}$ obtained at $8 \mathrm{~K}$ in the backscattering configuration from (112) plane for parallel polarization of the incident and scattered light. The excitation wavelength is $514.5 \mathrm{~nm}$ and spectral resolution $0.9 \mathrm{~cm}^{-1}$. Notice again the large intensity of the $\Gamma_{1}$ phonon mode at $317 \mathrm{~cm}^{-1}$. 


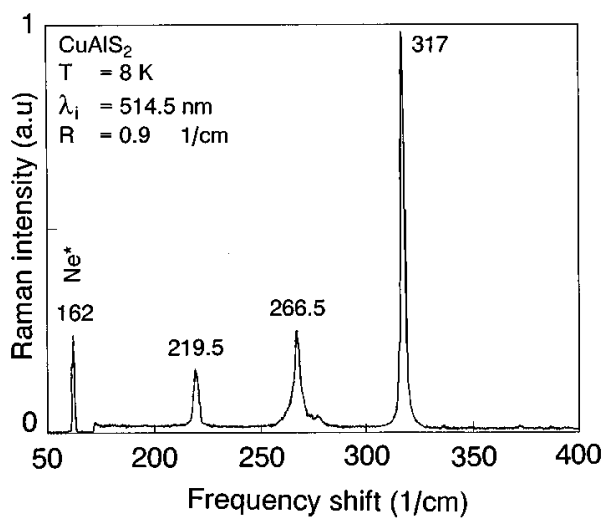

FIG. 5. Raman spectrum of $\mathrm{CuAlS}_{2}$ obtained at $8 \mathrm{~K}$ in backscattering configuration from (112) plane for parallel polarizations of incident and scattered light. The excitation wavelength is $514.5 \mathrm{~nm}$ and spectral resolution is $0.9 \mathrm{~cm}^{-1}$.

type semiconductors with folding of all Brillouin zone edge points labeled $X(0,0,2 \pi / a), W(0,2 \pi / a, \pi / a)$ and $W(2 \pi /$ $a, 0, \pi / a)$ back to the center of the Brillouin zone with zone center $(\Gamma$-like $)$ representations. Since the chalcopyrite structure compounds have two formula units with eight atoms per unit cell, giving rise to 21 optical and three acoustic branches of lattice vibrations, the representation, $\Gamma$ at the center of the zone, is reducible into

$$
\Gamma=1 \Gamma_{1}+2 \Gamma_{2}+3 \Gamma_{3}+3 \Gamma_{4}+6 \Gamma_{5} \text { and } 1 \Gamma_{4}+1 \Gamma_{5},
$$

respectively. With the splitting of infrared active modes by the macroscopic long range electrostatic interaction into transverse and longitudinal components as well as the $\Gamma_{4}$ and $\Gamma_{5}$ optical modes and since the $\Gamma_{2}$ modes are silent, one expects 22 Raman-active optical modes

$1 \Gamma_{1}+3 \Gamma_{3}+3 \Gamma_{4}(\mathrm{LO})+3 \Gamma_{4}(\mathrm{TO})+6 \Gamma_{5}(\mathrm{LO})+6 \Gamma_{5}(\mathrm{TO})$.

The symmetry types of the Raman-active modes for chalcopyrite-type structure crystals with corresponding zincblende structure modes are listed in Table I.

Previously, the vibrational spectra of $\mathrm{CuAlS}_{2}$ by zone center optical phonons have been investigated at room tem-

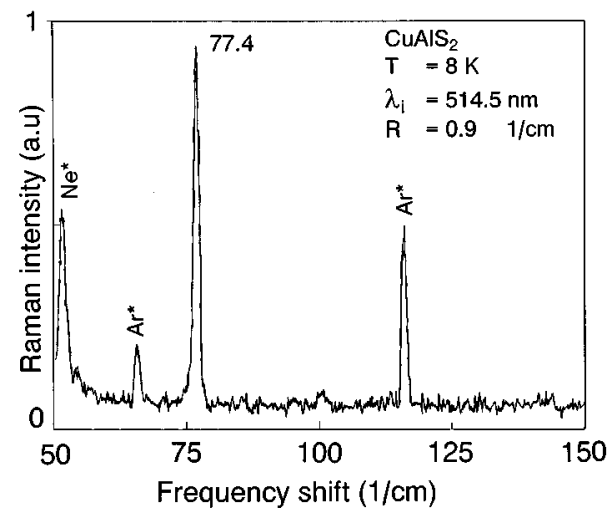

FIG. 6. Raman spectrum of $\mathrm{CuAlS}_{2}$ obtained at 300 and $8 \mathrm{~K}$ in backscattering configuration from (112) plane for crossed polarizations of incident and scattered light. Notice the $\Gamma_{5}(\mathrm{LO}, \mathrm{TO})$ phonon mode at $442.5 \mathrm{~cm}^{-1}$ and disappearance of second-order structure at $553 \mathrm{~cm}^{-1}$. The excitation wavelength is $514.5 \mathrm{~nm}$ and spectral resolution is $0.9 \mathrm{~cm}^{-1}$.

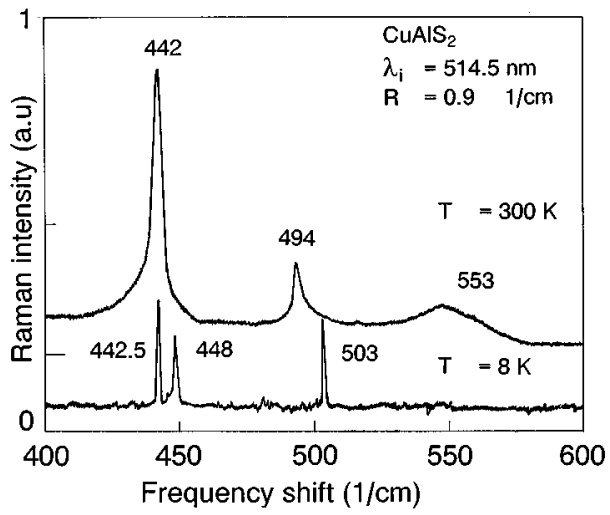

FIG. 7. Raman spectrum of $\mathrm{CuAlS}_{2}$ obtained at $8 \mathrm{~K}$ in backscattering configuration from (112) plane for parallel polarizations of incident and scattered light. The excitation wavelength is $514.5 \mathrm{~nm}$ and spectral resolution is $0.9 \mathrm{~cm}^{-1}$.

perature by Raman scattering as well as by infrared absorption and reflectivity measurements by Koshell et al. ${ }^{21,22}$ In addition to the already known phonon modes of $\mathrm{CuAlS}_{2}$ at $T=300 \mathrm{~K}$, we describe here corrections of previous measurements and assignments of newly observed lines.

Figure 1 shows the overall Raman scattering spectrum of $\mathrm{CuAlS}_{2}$ obtained at room temperature in the frequency range of $0-600 \mathrm{~cm}^{-1}$. The most interesting feature of this spectrum is the large intensity of the $\Gamma_{1}$ phonon mode at $314 \mathrm{~cm}^{-1}$. Other Raman lines are also revealed, but their intensities are several times smaller. Figures 2 and 3 show the Raman scattering spectra of those lines in expanded scales for frequency ranges of $150-400$ and $50-150 \mathrm{~cm}^{-1}$, respectively. One can easily see the appearance of new structures at 123,272 , and $372 \mathrm{~cm}^{-1}$.

In order to resolve the observed broad structures and to distinguish between first and second order scattering structures at $263,442.5$, and $553 \mathrm{~cm}^{-1}$, we have performed Raman measurements at $8 \mathrm{~K}$. The overall spectra obtained in

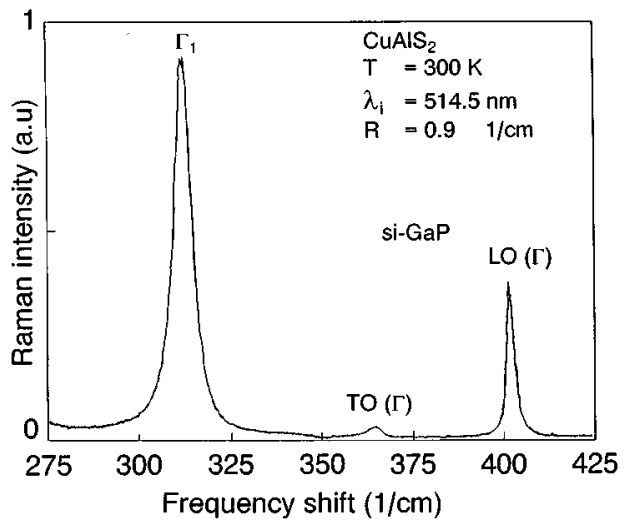

FIG. 8. Room temperature Raman spectrum of $\mathrm{CuAlS}_{2}$ obtained in the backscattering configuration from (112) plane for parallel polarization of the incident and scattered light and of si-GaP obtained also in the backscattering configuration from (111) plane for identical experimental conditions including the same incident power of $2 \mathrm{~mW}$ and detection intensity scale. Notice the large absolute scattering intensity of $\Gamma_{1}$ phonon mode at 314 $\mathrm{cm}^{-1}$ in comparison with $\mathrm{LO}(\Gamma)$ phonon mode at $402.3 \mathrm{~cm}^{-1}$ of $\mathrm{Si}-\mathrm{GaP}$. 
the range $0-600 \mathrm{~cm}^{-1}$ is given in Fig. 4, while the spectra obtained in expanded scales are presented in Figs. 5 and 6. With the same aim, we display in Fig. 7 the Raman spectra of $\mathrm{CuAlS}_{2}$ obtained at room temperature and $8 \mathrm{~K}$ in the range $400-600 \mathrm{~cm}^{-1}$.

In Table I we summarize the results of our measurements compared to previous Raman as well as infrared absorption, and reflectivity, measurements.

For the broad line observed at room temperature at 442 $\mathrm{cm}^{-1}$ with structures from both sides of this line, we have found at $8 \mathrm{~K}$ the appearance of two new lines centered at 442.5 and $448.5 \mathrm{~cm}^{-1}$, respectively. For the other broad line at $494 \mathrm{~cm}^{-1}$ at $T=300 \mathrm{~K}$ with asymmetric structure from the high frequency side we found a single sharp line at 503 $\mathrm{cm}^{-1}$. The other very broad structure at 553 (at $T=300 \mathrm{~K}$ ) disappeared at $8 \mathrm{~K}$.

It is interesting to note that the $\Gamma_{1}$ phonon mode always dominates the Raman spectra of all chalcopyrite structure semiconductors. ${ }^{19-25}$ It appears in the spectra for all scattering configurations used.

The high scattering intensity and narrow linewidth of the $\Gamma_{1}$ mode in $\mathrm{CuAlS}_{2}$ indicate a high value of gain for Raman oscillator operation in the process of stimulated Raman scattering.

The power gain coefficient $g_{s} / I$, where $I$ is the laser pump power per unit area for the stimulated Raman scattering and for the Stokes wave, was given by Shen and Blombergen. ${ }^{26}$ By taking into account corrections given by Johnston and Kaminow ${ }^{27}$

$$
\frac{g_{s}}{I}=\frac{16 \pi^{2} c^{2}}{h \omega_{s}^{3}} \frac{S / L d \Omega}{n_{i} n_{s}\left(n_{0}+1\right) \Gamma} .
$$

Here, $\omega_{s}$ is the frequency of the scattered light at the Stokes frequency, $n_{i}$ and $n_{s}$ are refractive indices at the incident laser and scattered Stokes frequencies, $n_{0}$ is the Bose population factor, $\left[\exp (h \omega / k T)^{-1}\right]^{-1}$, and $\Gamma$ is the linewidth full width at half maximum (FWHM) of a mode.

The power at the Stokes frequency increases with length $L$ as $\exp \left(g_{s} L\right)$. Thus available power gain for stimulated Raman scattering amplification can be obtained from the measurement of the absolute scattering efficiency. The strength of a given Raman-active phonon mode in the process of spontaneous scattering is given by the absolute scattering efficiency $S / L d \Omega$ where $S$ is the fraction of the incident laser power that is scattered into a solid angle of $d \Omega$ near a normal to the optical path $L$.

The efficiency of an unknown spectral mode can be obtained by comparison with integrated scattering intensities (areas under spectral lines) of measured and reference samples under identical experimental conditions.

To measure the absolute scattering efficiency of $\Gamma_{1}$ mode in $\mathrm{CuAlS}_{2}$, we used as reference the scattering efficiency of the $\mathrm{LO}(\Gamma)$ phonon line in semi-insulating $\mathrm{GaP}$ crystals (where plasmon-phonon interaction play no role). ${ }^{15}$

Figure 8 shows the room temperature Raman spectrum of $\mathrm{CuAlS}_{2}$ obtained in the backscattering configuration from (112) plane for parallel polarizations of incident and scattered light and for si-GaP obtained for identical experimental conditions. One can see the large absolute scattering ef- ficiency of $\Gamma_{1}$ phonon mode in $\mathrm{CuAlS}_{2}$ at $314 \mathrm{~cm}^{-1}$ in comparison with that of $[\mathrm{LO}(\Gamma)]$ phonon mode of si-GaP at $402.3 \mathrm{~cm}^{-1}$. The calibrated ratio of the areas under these two spectral lines is a factor of 7 . The value of $S / L d \Omega$ for si$\mathrm{GaP}$ was measured by using as a reference standard material benzene. For the $992 \mathrm{~cm}^{-1}$ mode of benzene, the absolute scattering efficiency $S / L d \Omega=1.0 \times 10^{-7} \mathrm{~cm}^{-1}$ at $488 \mathrm{~nm}$ has been determined by other means. ${ }^{27,28}$

As already mentioned, the high absolute scattering efficiency of $1.9 \times 10^{-5} \mathrm{~cm}^{-1} \mathrm{sr}^{-1}$ at $\lambda_{i}=632.8 \mathrm{~nm}$ for scattering by $\mathrm{LO}(\Gamma)$ phonons at $402.3 \mathrm{~cm}^{-1}$ observed in si-GaP together with the anomalously narrow linewidth of $0.6 \mathrm{~cm}^{-1}$ at room temperature indicated a large stimulated gain coefficient, ${ }^{15}$ namely $g_{s} / I=1.0 \times 10^{-6} \mathrm{~cm} / \mathrm{W}$. This is the highest gain for stimulated Raman amplification value measured so far for various materials (Table I) including the most efficient $\mathrm{Li}^{6} \mathrm{NbO}_{3}\left(\mathrm{LiNbO}_{3}\right.$ made with the rare $\mathrm{Li}^{6}$ isotope rather than the abundant $\left.(\sim 92.5 \%) \mathrm{Li}^{7}\right)$ (with $256 \mathrm{~cm}^{-1}$

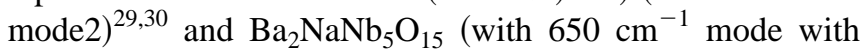
the highest absolute scattering efficiency but rather large linewidth ${ }^{31}$ of $28 \mathrm{~cm}^{-1}$ ).

Since the scattering efficiency of a given Raman-active mode $S / L d \Omega \sim \omega_{s}^{+}$, it is clear from (1) that the power gain coefficient $g_{s} / I$ is inversely proportional to the frequency of the scattered light $\omega_{s}$ (in frequency regions far from electronic resonance) and, therefore, may be taken as a basis for comparison of various promising materials.

The available quantitative data quoted for $\lambda_{i}=1.065 \mu \mathrm{m}$ excitation wavelength for appropriate vibrational modes of different materials of current and historic interest are listed in Table I. Due to the large power gain coefficient measured in si-GaP, the low threshold of pump laser power as a prerequisite for operation of a cw Raman oscillator on $\mathrm{GaP}$ has been predicted. ${ }^{15}$ Such a semiconductor laser can be pumped with laser diode arrays.

Recently, the low threshold-power operation of a buried heterostructure Raman laser based on GaP active layer and $\mathrm{Al}_{x} \mathrm{Ga}_{1-x} \mathrm{P}$ cladding layers, grown by the temperature difference method under controlled vapor pressure, has been demonstrated. ${ }^{16}$ The threshold pump power was as small as $500 \mathrm{~mW}$. Furthermore, by developing a tapered waveguide structure of growth $\mathrm{GaP}$ core and $\mathrm{Al}_{x} \mathrm{Ga}_{1-x} \mathrm{P}$ cladding layers and thus increasing the internal pump power, it became possible $^{17}$ to reduce the threshold of pump power down to $160 \mathrm{~mW}$.

After taking into account the scattering geometry and corresponding parameters for reference si-GaP and our $\mathrm{CuAlS}_{2}$ crystals for $\lambda_{i}=514.5 \mathrm{~nm}$ excitation and by using measurement techniques described in Refs. 15 and 29, we find for the most intense $\Gamma_{1}$ phonon mode at $314 \mathrm{~cm}^{-1}$ of $\mathrm{CuAlS}_{2}$ the absolute scattering efficiency for spontaneous Raman scattering $S / L d \Omega=9.5 \times 10^{-5} \mathrm{~cm}^{-1} \mathrm{sr}^{-1}$ and linewidth (FWHM) of $\Gamma=5.1 \mathrm{~cm}^{-1}$ at room temperature. For comparison, the large value of absolute scattering efficiency of $2.3 \times 10^{-6} \mathrm{~cm}^{-1} \mathrm{sr}^{-1}\left(\lambda_{i}=514.5 \mathrm{~nm}\right)$ for $\mathrm{LO}(\Gamma)$ phonons in GaAs at room temperature for $1.065 \mu \mathrm{m}$ excitation laser wavelength using the same measurement procedure has been reported. ${ }^{29}$

From Eq. (2) we calculate the corresponding power gain 
coefficient for stimulated Raman scattering process as $g_{s} / I=2.1 \times 10^{-6} \mathrm{~cm} / \mathrm{W}$. It is interesting to note that the absolute scattering efficiency normalized by the phonon population factor exhibits relatively small variation by reducing the temperature down to $8 \mathrm{~K}$. On the other hand, in this temperature range, the linewidth decreases substantially due to anharmonic interaction, while the frequency of the $\Gamma_{1}$ mode shifts to $317 \mathrm{~cm}^{-1}$. Cooling of $\mathrm{CuAlS}_{2}$ reduces the linewidth of this $\Gamma_{1}$ mode to $1.5 \mathrm{~cm}^{-1}$ at $8 \mathrm{~K}$ with corresponding increase of power gain coefficient $g_{s} / I=5.0 \times 10^{-6}$ $\mathrm{cm} / \mathrm{W}$ (for excitation wavelength $\lambda_{i}=514.5 \mathrm{~nm}$ ). The results given in Table I show that chalcopyrite structure $\mathrm{CuAlS}_{2}$ crystal for $314 \mathrm{~cm}^{-1} \Gamma_{1}$ mode has the largest power gain coefficient measured so far. Estimations for such large nonlinearity for stimulated Raman amplification show that a cw or quasi-cw Raman oscillator operation with $314 \mathrm{~cm}^{-1} \Gamma_{1}$ mode of $\mathrm{CuAlS}_{2}$ is feasible with low power threshold of pump power.

\section{CONCLUSIONS}

We have examined the spontaneous Raman spectra for nonlinear applications of promising chalcopyrite structure $\mathrm{CuAlS}_{2}$ crystals at 300 and $8 \mathrm{~K}$ with the main aim to find the relationship between the absolute Raman scattering intensities of $\mathrm{CuAlS}_{2}$ and their isomorphous analog zinc-blende structure $\mathrm{GaP}$ (known as one of the most efficient materials for stimulated Raman scattering amplification). The observed large absolute Raman scattering efficiency $S / L d \Omega=9.5 \times 10^{-6} \mathrm{~cm}^{-1} \mathrm{sr}^{-1}$ for laser excitation at 514.5 $\mathrm{nm}$ of the most intense $314 \mathrm{~cm}^{-1} \Gamma_{1}$ mode together with narrow linewidth of $5.1 \mathrm{~cm}^{-1}$ at room temperature and 1.5 $\mathrm{cm}^{-1}$ at $8 \mathrm{~K}$, indicates that $\mathrm{CuAlS}_{2}$ has a large value of power gain coefficient for stimulated Raman amplification. The calculated value of this gain reaches a practically attractive level $g_{s} / I=2.1 \times 10^{-6} \mathrm{~cm} / \mathrm{W}$ at $300 \mathrm{~K}$ and $5 \times 10^{-6}$ $\mathrm{cm} / \mathrm{W}$ at $8 \mathrm{~K}$, which are larger than those for the appropriate vibrational modes of various materials, including $\mathrm{GaP}$, $\mathrm{LiNbO}_{3}, \mathrm{Ba}_{2} \mathrm{NaNb}_{5} \mathrm{O}_{15}$, and $\mathrm{CS}_{2}$, investigated so far. Observation of such a large gain should permit $\mathrm{cw}$ or quasi-cw stimulated Raman amplification operation at room temperature with low power threshold.

\section{ACKNOWLEDGMENTS}

The authors would like to thank D. Vardar and M. Atature for technical assistance. One of us (B.H.B) would like to thank the Turkish Scientific and Technical Research Council (TUBITAK) for financial support under the DOPROG program during his stay at Bilkent.
${ }^{1}$ J. L. Shay and H. M. Wernick, Ternary Chalcopyrite Semiconductors, Growth, Electric Properties and Applications, edited by B. R. Pamplin (Pergamon, New York, 1979).

${ }^{2}$ N. C. Giles, L. E. Halliburton, P. G. Schunemann, and T. M. Pollak, Appl. Phys. Lett. 66, 1758 (1995).

${ }^{3}$ M. H. Rakowsky, W. K. Kuhn, W. J. Lauderdale, L. E. Halliburton, G. J. Edwards, M. P. Scriptek, P. G. Schunemann, T. M. Pollak, M. C. Ohmer, and F. K. Hopkins, Appl. Phys. Lett. 64, 1615 (1994).

${ }^{4}$ P. A. Budni, P. G. Schunemann, M. G. Knights, T. M. Pollak, and E. P. Chicklis, OSA Proceedings on Advanced Solid State Lasers, edited by L. Chase and A. A. Pinto (Optical Society of America, Washington DC, 1992), Vol. 13, pp. 380-383.

${ }^{5}$ G. D. Boyd, H. M. Kasfer, J. H. McFee, and F. G. Storz, IEEE J. Quantum Electron. QE-8, 900 (1972).

${ }^{6}$ B. Tell, J. L. Shay, and H. M. Kasper, Phys. Rev. B 4, 2463 (1971).

${ }^{7}$ W. Horig, H. Neuman, H. Sobota, B. Schumann, and G. Kuhn, Thin Solid Films 48, 67 (1978)

${ }^{8}$ L. L. Kazmerski, Nuovo Cimento D2, 2013 (1983).

${ }^{9}$ E. Jaffe and A. Zunger, Phys. Rev. B 29, 1882 (1984).

${ }^{10}$ I. Aksenov, T. Yasuda, Y. Segawa, and K. Sato, J. Appl. Phys. 74, 2106 (1993).

${ }^{11}$ K. Hara, T. Shinozawa, J. Yoshino, and H. Kukimoto, J. Cryst. Growth 93, 771 (1988).

${ }^{12}$ Y. Morita and T. Narusawa, Jpn. J. Appl. Phys. 31, 11396 (1992).

${ }^{13}$ R. Loudon, Adv. Phys. 13, 423 (1964).

${ }^{14}$ B. H. Bairamov, Yu. E. Kitaev, V. K. Nogoduyko, and Z. M. Khashkhozev, Sov. Phys. Solid State 16, 225 (1974).

${ }^{15}$ B. H. Bairamov, V. D. Timofeev, V. V. Toporov, and S. B. Ubaidullaev, Sov. Phys. Solid State 20, 1916 (1978).

${ }^{16}$ K. Suto, S. Ogasawara, T. Kimura, and J. Nichizawa, J. Appl. Phys. 66, 5151 (1989).

${ }^{17}$ K. Suto, T. Kimura, and J. Nishizawa, Int. J. Infrared Millim. Waves 16, 691 (1995).

${ }^{18}$ G. Brandt, A. Rauber, and J. Schneider, Solid State Commun. 12, 481 (1973).

${ }^{19}$ H. Tanino, T. Maeda, H. Fujikake, and H. Nananishi, Phys. Rev. B 45, 13323 (1992).

${ }^{20}$ J. Camassel, L. Artus, and J. Pascual, Phys. Rev. B 41, 5717 (1990).

${ }^{21}$ W. H. Koschell, W. Hohler, A. Rauber, and J. Baars, Solid State Commun. 13, 1011 (1973).

${ }^{22}$ W. H. Koschell and M. Bettini, Phys. Status Solidi B 72, 729 (1975).

${ }^{23}$ J. P. van der Zeil, A. E. Meixner, H. M. Kasper, and J. Ditzenberger, Phys. Rev. B 9, 4286 (1974).

${ }^{24}$ C. Carlone, D. Olego, A. Jayaraman, and M. Cardona, Phys. Rev. B 22, 3877 (1980).

${ }^{25}$ J. Gonzalez, B. J. Fernandez, J. M. Besson, M. Gauthier, and M. Polian, Phys. Rev. B 46, 15092 (1992).

${ }^{26}$ Y. R. Shen and N. Blombergen, Phys. Rev. 137A, 187 (1965).

${ }^{27}$ W. D. Johnston, Jr. and I. P. Kaminow, Phys. Rev. 168, 1045 (1968); 178, 1528E (1968).

${ }^{28}$ J. G. Skinner and W. G. Nilsen, J. Opt. Soc. Am. 58, 113 (1968).

${ }^{29}$ W. D. Johnston, Jr. and I. P. Kaminow, Phys. Rev. 188, 1209 (1969).

${ }^{30}$ W. Johnston, Jr., I. P. Kaminow, and J. G. Bergman, Jr., Appl. Phys. Lett. 13, 1980 (1968).

${ }^{31}$ M. K. Srivastava and R. W. Crow, Opt. Commun. 8, 82 (1973).

${ }^{32}$ E. P. Ippen, Appl. Phys. Lett. 16, 330 (1970).

${ }^{33}$ N. Blombergen, G. Bret, P. Lallemand, A. Pine, and P. Simova, IEEE J. Quantum Electron. 3, 197 (1967). 